\title{
Structure des troupeaux et performances des élevages porcins de la zone de N'Djaména au Tchad
}

\author{
L. Y. MOPATE ${ }^{1 *}$, C. Y. KABORE-ZOUNGRANA ${ }^{2}$ et B. FACHO ${ }^{3}$ \\ ${ }^{1}$ Laboratoire de Recherches Vétérinaires et Zootechniques de Farcha (LRVZ), B. P. 433 N’Djaména (Tchad). \\ Tel. / Fax (00235) 527877 ; \\ ${ }^{2}$ Laboratoire d'Etudes et de Recherches des Ressources Naturelles et des Sciences de l'Environnement \\ (LERNSE), Université Polytechnique de Bobo-Dioulasso (UPB) au Burkina Faso, 01 B.P. 1091 Bobo- \\ Dioulasso 01, Tel. (00226) 209806 35,E-mail : cykabore@yahoo.fr \\ ${ }^{3}$ Faculté des Sciences Exactes et Appliquées (FSEA), Université de N'Djaména, B. P. 1027, N'Djaména, \\ Tchad, E-mail :facho_balaam@yahoo.fr \\ "Auteur correspondant,E-mail : mopate.ly@gmail.com ; mopate_ly@yahoo.fr
}

\section{RESUME}

L'objet du travail a été de déterminer la structure des troupeaux et d'évaluer les performances des élevages porcins de la zone de N'Djaména. Elle a été réalisée par enquête transversale et rétrospective et par enquête longitudinale sur un échantillon de 176 éleveurs. En ville, 4 des 5 arrondissements producteurs de porcs, 8 quartiers, et à la périphérie, 12 villages tirés au hasard ont été concernés. Le nombre d'éleveurs enquêtés représentait $12 \%$ des éleveurs en ville et $20 \%$ à la périphérie. Pour l'enquête longitudinale, 15 élevages dont 8 en ville et 7 à la périphérie ont été suivis pendant un an. Dans cette zone, les troupeaux ont été composés de $60 \%$ de femelles et $40 \%$ de mâles. L'âge moyen à la $1^{\text {ère }}$ mise-bas (MB) a été de 10,90 $\pm 1,90$ mois. Le nombre moyen de MB par an a été de 1,98 \pm 0,35, la portée moyenne a été de 15,94 \pm 4,97 porcelets/truie/an et la productivité numérique de 13. Le revenu moyen brut par an a été de $247780 \mathrm{~F}$ CFA en ville et $123570 \mathrm{~F}$ à la périphérie. Les performances de reproduction ont été relativement meilleures comparées à celles des élevages ruraux. Les performances zootechniques et socio-économiques obtenues constituent une base pour l'évaluation des interventions futures.

(c) 2011 International Formulae Group. All rights reserved.

Mots Clés : Porc, composition des troupeaux, performances zootechniques, performances socio-économiques, N'Djaména, Tchad.

\section{INTRODUCTION}

Les villes et leurs périphéries constituent des centres de productions agropastorales, dont la contribution dans l'alimentation des citadins est indéniable (Moustier et De Bon, 2005). Les villes représentent aussi des débouchés sûrs pour les produits agricoles des zones proches et éloignées (Guérin et Faye, 1999). Ainsi, l'analyse des dynamiques agricoles et des performances des filières face aux nouvelles opportunités et aux contraintes inhérentes à la proximité de la ville est nécessaire pour mieux les apprécier (Broutin et al., 2005).

N'Djamena et sa périphérie constituent le deuxième bassin de production de porcs au Tchad, après la zone soudanienne (Mopaté, 2008). La population de N'Djaména est passée de 530965 habitants en 1993 à 993492 personnes en 2009 (MEP, 2009), soit 
une croissance annuelle de 5,4\%. Cette forte urbanisation de la ville n'est pas sans conséquence, sur l'approvisionnement en denrées animales des citadins. A la faveur de l'accroissement de la demande externe et interne en viande porcine (Mopaté et al., 2008), un développement des élevages à N'Djaména est observé au cours de deux dernières décennies (Mopaté et al., 2007). Ces observations impliquent la connaissance de la structure des troupeaux et des performances de la production des porcs dans et autour de la ville de N'Djaména.

L'objet de l'étude a été de déterminer la structure des troupeaux et d'évaluer les performances zootechniques et socioéconomiques des élevages porcins de la zone de N'Djaména.

\section{MATERIEL ET METHODES Site d'étude}

L'étude a été réalisée dans les zones urbaine et périurbaine de la ville de N'Djaména, capitale du Tchad. Les coordonnées géographiques de cette ville, relevées par Global Positioning System (GPS) sont $12^{\circ} 11^{\prime} 30^{\prime}$ ' de latitude Nord et $15^{\circ} 04^{\prime}$ 91', de longitude Est.

\section{Echantillonnage et méthode de collecte des données}

L'enquête transversale et rétrospective a été réalisée dans 4 des 5 arrondissements producteurs de porcs $\left(1^{\mathrm{er}}, 3^{\mathrm{e}}, 7^{\mathrm{e}}\right.$ et $\left.9^{\grave{e}}\right)$ à N'Djaména qui ont été tirés au hasard. Il en a été de même des quartiers enquêtés : Farcha et Madjorio pour le $1^{\mathrm{er}}$ arrondissement, Sabangali pour le $3^{\text {è }}$, Chagoua, Dembé, Abena et Atrone pour le $7^{\mathrm{e}}$, Karway et Walia pour le $9^{\mathrm{e}}$. Le nombre d'éleveurs enquêté correspondait à $12 \%$ des éleveurs en zone urbaine. Dans la zone périurbaine (rayon de $100 \mathrm{Km}$ ), 12 villages dont 9 à la périphérie sud et 2 dans la partie nord ont été également choisis de manière aléatoire. En raison des effectifs moins importants de producteurs comparés à la zone urbaine, 20\% des éleveurs ont été enquêtés. Au total 176 éleveurs ont été échantillonnés et enquêtés dans les deux zones.
Les points abordés ont été les effectifs et la composition des troupeaux, les paramètres de reproduction et les performances socio-économiques (objectifs de production, exploitation, utilisation des recettes et les motifs de consommation).

La seconde phase a été le suivi de 15 élevages porcins dont 8 en ville et 7 à la périphérie. Au total, 287 animaux dont 168 en ville et 119 à la périphérie ont été identifiés individuellement à l'aide des boucles auriculaires numérotées. Le rythme de passage mensuel dans les élevages a permis d'enregistrer les données de reproduction, le poids de la portée à la mise-bas et la croissance pondérale. Le suivi pondéral a été effectué jusqu'à l'âge de 12 mois pour les mâles et 7 mois seulement pour les femelles afin d'éviter le fait biais possible dû à une gestion.

\section{Analyse des données}

Les saisies ont été effectuées avec le logiciel de gestion de base des données «Access », puis transférées dans le logiciel de traitement des données «Winstat-ic» du Cirad. Les variables caractères ont été recodées en variables numériques qualitatives avec des modalités. Des regroupements de certaines modalités ont été effectués. Un tri à plat a été effectué pour opérer un choix des variables qualitatives et quantitatives à soumettre en analyse approfondie. Le couple des variables sexe et âge des animaux enquêtés a été considéré pour établir la structure des troupeaux. Pour l'analyse de variance, la ventilation des performances zootechniques et socio-économiques moyennes a été faite sur le facteur zone (urbaine ou périurbaine). Dans les élevages suivis, la taille moyenne des portées a été déterminée selon le rang de mise-bas. L'évolution pondérale mensuelle a été établie selon le sexe des animaux. Les moyennes ont été données avec des écart-types. Un seuil de signification de $5 \%$ a été retenu.

L'âge à la $1^{\text {ère }}$ gestation a été déduit à partir de l'âge à la $1^{\text {ère }}$ mise-bas par soustraction de 4 mois environ de gestation. Le revenu moyen brut issu de cet élevage par 
an est égal à la moyenne des animaux vendus et consommés par an, multiplié par le prix moyen. Ce revenu brut a été divisé par le nombre de ménages pour avoir la moyenne.

\section{RESULTATS}

Les éleveurs échantillonnés ont été repartis pour $59 \%$ en ville et $41 \%$ à la périphérie. Leur âge moyen a été de 43,76 \pm 13,73 ans. Ils sont producteurs agricoles $(52 \%)$, salariés $(24 \%)$, petits commerçants, ouvriers, manœuvres et artisans (11\%), étudiants et élèves $(9 \%)$ et retraités $(4 \%)$. La majorité des producteurs urbains $(75 \%$ des hommes et $87 \%$ des femmes) s'est lancée dans cette production dans les années 2000 et $16 \%$ dans la décennie 1990. En zone périurbaine, ils ont été $56 \%$ à démarrer dans la décennie 2000 et $33 \%$ dans les années 1990.

\section{Structure des troupeaux}

Sur 3392 porcs de l'échantillon, 63\% ont été dénombrés en ville contre $27 \%$ à la périphérie. Le troupeau moyen échantillonné dans la zone de N'Djaména a été de 19,27 \pm 14,55 porcs. La différence des moyennes entre la ville $(20,68 \pm 16,01)$ et la périphérie $(17,27$ $\pm 12,03)$ n'a pas été significative $(\mathrm{P}>0,05)$. Les troupeaux ont été composés de $60 \%$ de femelles et $40 \%$ de mâles, sans différence entre la ville et sa périphérie. Les jeunes de 0 à 7 mois ont constitué $71 \%$ des effectifs (Tableau 1), avec un mâle pour 1,5 femelles, mais ce rapport passe à 2,4 à partir de 8 mois d'âge. La pyramide des âges (Figure 1) a mis en évidence la chute des effectifs mâles dans les élevages après 7 mois. Ce phénomène a été davantage marqué après 12 mois. En tout, $18,8 \%$ des mâles ont été castrés dans $45 \%$ des élevages à l'âge de 7 mois. Cet âge a été moins élevé $(\mathrm{P}<0,05)$ en ville $(6,56 \pm 3,59)$ qu'à la périphérie $(8,70 \pm 5,17)$.

\section{Performances zootechniques des élevages Performances de reproduction des porcs de race locale}

L'âge à la $1^{\text {ère }}$ mise-bas $(\mathrm{MB})$ a été de 11 mois et la $1^{\text {ère }}$ saillie fécondante de 7 mois (gestation estimée à 4 mois environ). Le nombre de MB moyen par an, la taille de portée moyenne par truie par an et l'âge moyen au sevrage ont été plus élevés ( $\mathrm{P}<$ 0,05 ) en ville qu'à la périphérie (Tableau 2)

Dans 40 élevages qui ont déclaré des avortements, $63 \%$ ont été en ville. Les fréquences moyennes des avortements ont été 2 fois plus élevées en ville qu'à à la périphérie $(\mathrm{P}<0,05)$. Les truies qui ont avorté ont été soit réformées (48\%), soit traitées $(37 \%)$ ou laissées à leur sort (15\%). L'essentiel des traitements a été effectué en ville.

La majorité des éleveurs (84\%) ont réformé des verrats à un âge moyen de 2,22 \pm 0,76 ans et $81 \%$ des truies à $3,15 \pm 1,29$ ans en moyenne. L'age moyen des truies a été significativement plus élevé $(\mathrm{P}<0,001)$ en ville $(3,52 \pm 1,37)$ qu'à la périphérie $(2,63 \pm$ $0,96)$.

Dans les élevages suivis, 55 mises-bas (MB) ont été enregistrées. Elles ont donné 436 porcelets. La taille moyenne des portées a été de $7,93 \pm 1,95$ avec $47,5 \%$ des mâles contre $52,5 \%$ des femelles. Elle a augmenté régulièrement du $1^{\text {er }}$ au $4^{\text {ème }}$ rang de mise-bas (RMB). Le $4^{\text {ème }} \mathrm{RMB}$ a donné une portée moyenne plus élevée (Tableau 3).

La majorité des MB (60\%) sont intervenues durant la saison sèche. La première $\mathrm{MB}$ est survenue à 11,03 mois d'âge, la deuxième à 18,30 mois, soit un intervalle entre deux mises-bas (IMB) de 7,3 mois. La portée moyenne à la $\mathrm{MB}$ d'environ 8 porcelets a pesé $8,20 \mathrm{~kg}$, soit un poids moyen à la misebas des porcelets de $1,03 \mathrm{~kg}$.

\section{Performances pondérales des porcs de race locale}

La croissance a été relativement régulière de la naissance à 12 mois chez les mâles et 7 mois pour les femelles (Figure 2). Le gain de poids moyen quotidien (GMQ) entre 0 et 6 mois a été de $87,2 \mathrm{~g} / \mathrm{j}$ chez les mâles et de $91,5 \mathrm{~g} / \mathrm{j}$ chez les femelles.

Dans les deux sexes, les GMQ mensuels ont été variables de $102,33 \mathrm{~g}$ au $1^{\text {er }}$ mois, 96,66 $\mathrm{g}$ au $2^{\text {ème }}$ et 76,66 $\mathrm{g}$ au $3^{\text {ème }}$ mois chez les mâles. Chez les femelles, ils ont été de $103,33 \mathrm{~g}$ au $1^{\text {er }}$ mois, $94,00 \mathrm{~g}$ au $2^{\text {ème }}$ mois et $88,66 \mathrm{~g}$ au $3^{\text {ème }}$ mois. 


\section{Performances socio-économiques}

La vente et la consommation ont été à la fois les objectifs de production de $69 \%$ des producteurs en ville et $77 \%$ à la périphérie. Pour la zone de N'Djaména, en moyenne $73 \%$ ont affiché ces deux objectifs, $17 \%$ la vente exclusive et $10 \%$ la consommation seule. En ville, $95 \%$ de éleveurs ont vendu 858 porcs pour l'année soit en moyenne 9 porcs par élevage. A la périphérie, $97 \%$ ont écoulé 418 têtes, soit une moyenne de 6 têtes par élevage. Ces effectifs moyens ont été différents $(\mathrm{P}<$ $0,01)$ selon les zones. Environ 83\% des éleveurs urbains ont consommé en moyenne 4 porcs/an. A la périphérie, ils ont été $93 \%$ à en consommer 3 têtes en moyenne. Le taux d'exploitation a été de $55 \%$ en ville et $48 \%$ à la périphérie, soit $52,7 \%$ pour l'ensemble de la zone de N'Djaména.

Dans la catégorie de 8 mois et au-delà, l'âge et les prix moyens (Tableau 4) ont été plus élevés en ville qu'à la périphérie $(\mathrm{P}<$ $0,05)$. Pour les catégories d'âge inférieur, seul l'âge moyen à la vente entre la ville et la périphérie a varié $(\mathrm{P}<0,05)$.

Au prix moyen sans distinction d'âge de 19060 F CFA en ville, les éleveurs urbains ont gagné en moyenne, 171540 F/an. Ceux de la périphérie, $82380 \mathrm{~F}$ CFA pour leur vente au prix moyen de $13730 \mathrm{~F}$ CFA. Le revenu moyen brut par an et par élevage issu de la vente et de la consommation à la fois a été en moyenne de $247780 \mathrm{~F}$ en ville et de 123570 $\mathrm{F}$ à la périphérie. Au prix moyen du porc sans distinction d'âge et de zone d'environ $16400 \mathrm{~F}$ CFA, ces ventes (1 276) ont ainsi rapporté environ $21000000 \mathrm{~F}$ CFA aux éleveurs. Ces recettes sont allées surtout à l'achat des céréales (80\%), le reste à l'habillement (7\%), aux soins médicaux et la scolarité des enfants (5\%), aux achats de matériels agricoles, autres animaux et la paye de la main d'œuvre (3\%), à la construction des habitations, achat d'aliments pour porcs et entretien des porcheries (3\%), enfin aux autres biens de consommation (2\%).

Les motifs de consommation au foyer ont été l'accueil d'un hôte (37\%), la consommation ordinaire (32\%), les cérémonies $(23 \%)$, les sacrifices $(5 \%)$ et les travaux de groupe (3\%).

Dans les élevages suivis, $58 \%$ des recettes de ventes (environ $1115000 \mathrm{~F}$ ) sont allées à l'approvisionnement en céréales des éleveurs, $22 \%$ aux soins médicaux, à la scolarité et à l'habillement, $12 \%$ aux cérémonies festives et funérailles, $6 \%$ à l'entretien des porcheries, l'alimentation des porcs, l'équipement et la main d'œuvre, et $2 \%$ aux travaux champêtres et à l'achat d'autres animaux.

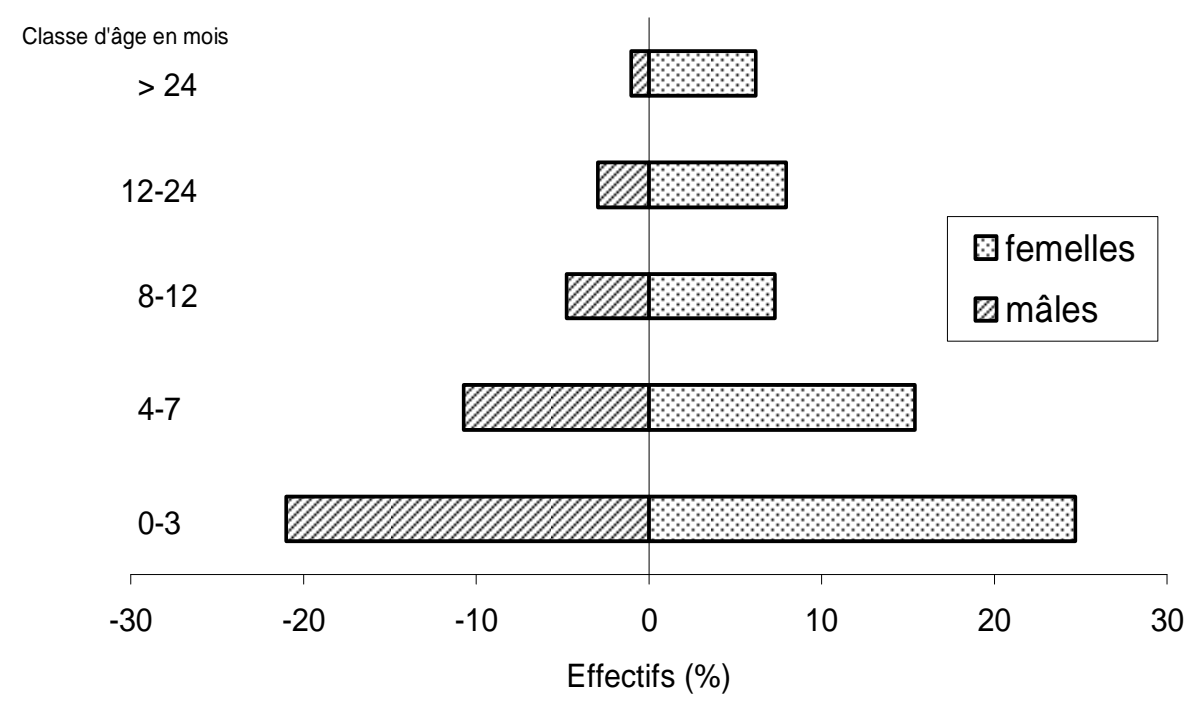

Figure 1 : Pyramide des âges des effectifs enquêtés dans la zone de N'Djaména (Tchad). 


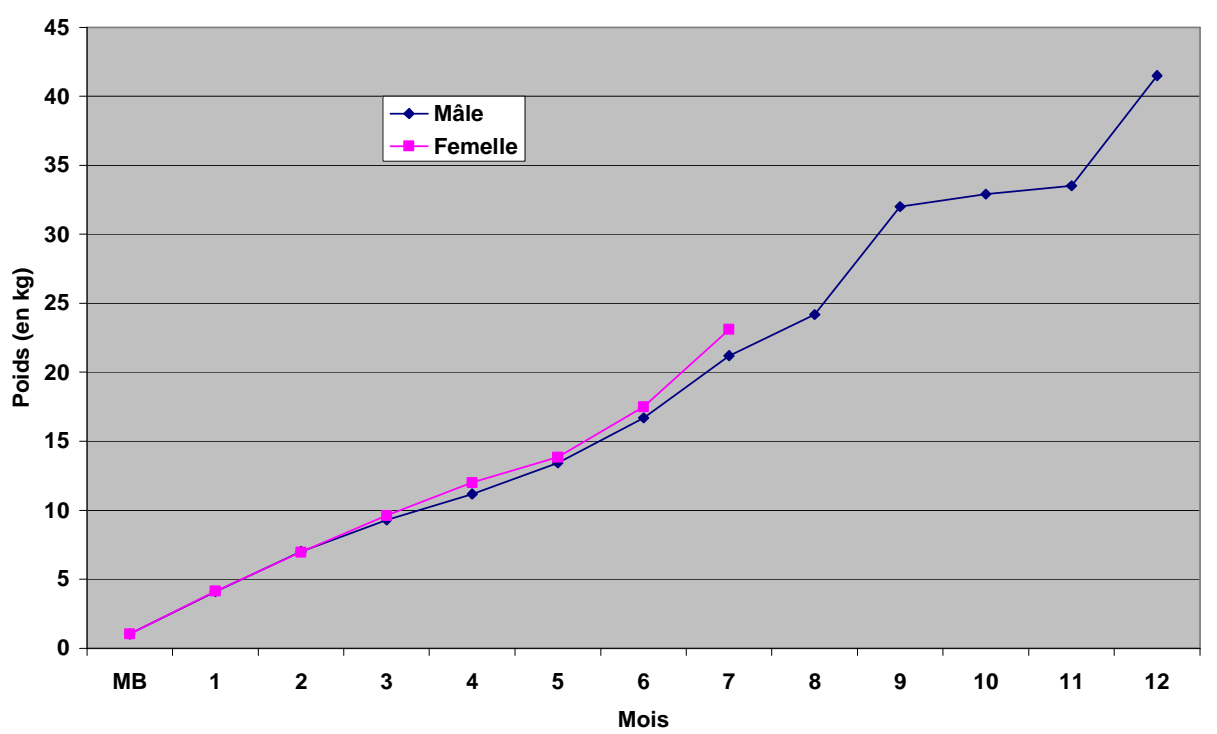

Figure 2 : Evolution pondérale des porcs en élevage familial de la zone de N’Djaména (Tchad). $\mathrm{MB}=$ mise bas.

Tableau 1 : Effectifs par classe d'âge (en mois) par sexe et par zone, du cheptel porcin enquêté dans la zone de N'Djaména (Tchad).

\begin{tabular}{|c|c|c|c|c|c|c|c|}
\hline \multirow{2}{*}{$\begin{array}{l}\text { Classe } \\
\text { d'âge } \\
\text { (mois) }\end{array}$} & \multicolumn{2}{|c|}{ Zone urbaine } & \multicolumn{2}{|c|}{ Zone périurbaine } & \multicolumn{2}{|c|}{ Total zone N'Djaména } & \multirow{2}{*}{$\begin{array}{l}(\%) / \\
\text { classe }\end{array}$} \\
\hline & Mâle & femelle & Mâle & Femelle & Mâle & Femelle & \\
\hline $0-3$ & 442 & 493 & 264 & 353 & 706 & 846 & 46 \\
\hline $4-7$ & 251 & 310 & 126 & 182 & 373 & 492 & 25 \\
\hline $8-12$ & 105 & 147 & 68 & 90 & 173 & 237 & 12 \\
\hline $13-24$ & 60 & 174 & 30 & 97 & 90 & 271 & 11 \\
\hline$>24$ & 20 & 128 & 2 & 54 & 22 & 182 & 06 \\
\hline Total /sexe & 878 & 1252 & 486 & 776 & 1364 & 2028 & \\
\hline Total /zone & \multicolumn{2}{|c|}{2130} & \multicolumn{2}{|c|}{1262} & \multicolumn{2}{|c|}{3392} & 100 \\
\hline
\end{tabular}

Tableau 2 : Performances de reproduction des élevages porcins de la zone de N'Djaména (Tchad).

\begin{tabular}{lccc}
\hline Paramètres de reproduction & Zone urbaine & Zone périurbaine & Zone N'Djaména \\
\hline Age (mois) $1^{\text {ère }}$ mise-bas & $10,9 \pm 2,3 \mathrm{a}$ & $11,04 \pm 1,03 \mathrm{a}$ & $10,9 \pm 1,9$ \\
Age (mois) $1^{\text {ère }}$ gestation & 7 & 7 & 7 \\
Nbre mise-bas/an & $2,03 \pm 0,38 \mathrm{a}$ & $1,92 \pm 0,28 \mathrm{~b}$ & $1,98 \pm 0,35$ \\
Taille de portée/an/truie & $16,51 \pm 5,15 \mathrm{a}$ & $15,15 \pm 4,64 \mathrm{~b}$ & $15,94 \pm 4,97$ \\
Taille portée nés-vivants/an/truie & $14,77 \pm 4,68 \mathrm{a}$ & $14,44 \pm 4,62 \mathrm{a}$ & $14,63 \pm 4,65$ \\
Taille portée sevrée/an/truies & $13,03 \pm 4,56 \mathrm{a}$ & $13,12 \pm 4,88 \mathrm{a}$ & $13,07 \pm 4,68$ \\
Age (mois) au sevrage & $3,85 \pm 1,10 \mathrm{a}$ & $3,11 \pm 0,52 \mathrm{~b}$ & $3,50 \pm 1,02$ \\
Avortement moyen /élevage & $1,68 \pm 1,0 \mathrm{a}(\mathrm{n}=25)$ & $1,40 \pm 0,74 \mathrm{~b}(\mathrm{n}=15)$ & $1,57 \pm 0,91(\mathrm{n}=40)$ \\
Mortinatalité/an & $10,5 \%(\mathrm{n}=68)$ & $6,2 \%(\mathrm{n}=27)$ & $8,4 \%(\mathrm{n}=95)$ \\
\hline \multicolumn{2}{c}{ Les moyennes des lignes (zones urbaine et périurbaine) suivies des lettres différentes, diffèrent significativement au seuil de } \\
5\%.
\end{tabular}


Tableau 3 : Variation de la taille de portée selon les rangs mise-bas des truies dans les élevages de la zone de N'Djaména (Tchad).

\begin{tabular}{lcccc}
\hline Effectif truie et & \multicolumn{4}{c}{ Rang de mise-bas } \\
\cline { 2 - 5 } taille portée & $1^{\text {er }}$ & $2^{\text {eme }}$ & $3^{\text {eme }}$ & $4^{\text {eme }}$ \\
\hline Nombre truie & 21 & 16 & 10 & 8 \\
Taille portée & $6,33 \pm 1,11$ a & $7,75 \pm 1,13 \mathrm{~b}$ & $9,20 \pm 0,63 \mathrm{c}$ & $10,88 \pm 1,64 \mathrm{~d}$ \\
\hline Les moyennes en ligne suivies des lettres différentes, diffèrent significativement au seuil de 5\%.
\end{tabular}

Tableau 4 : Variation des prix en fonction des âges (en mois) dans la zone de N’Djaména (Tchad).

\begin{tabular}{lcccc}
\hline \multirow{2}{*}{ Zone } & \multicolumn{2}{c}{ Porcs $\leq \mathbf{7}$ mois } & \multicolumn{2}{c}{ Porcs $\geq \mathbf{8}$ mois } \\
\cline { 2 - 5 } & Age moyen à la & Prix moyen de & Age moyen à la & Prix moyen de \\
& vente (mois) & vente (F CFA) & vente (mois) & vente (F CFA) \\
\hline Urbaine & $5,67 \pm 1,40 \mathrm{a}$ & $9300 \pm 3400 \mathrm{a}$ & $23,07 \pm 8,47 \mathrm{a}$ & $28820 \pm 7840 \mathrm{a}$ \\
Périurbaine & $6,76 \pm 1,20 \mathrm{~b}$ & $8800 \pm 2500 \mathrm{a}$ & $16,38 \pm 8,01 \mathrm{~b}$ & $18660 \pm 6135 \mathrm{~b}$ \\
Total & $6,12 \pm 1,37$ & $9050 \pm 2950$ & $20,45 \pm 8,89$ & $24840 \pm 8750$ \\
\hline Les moyennes des colonnes suivies des lettres différentes, differrent significativement au seuil de 5\%.
\end{tabular}

\section{DISCUSSION}

L'étude a permis de caractériser la structure des troupeaux et les performances de la production de porcs dans la zone de N'Djaména. Ces résultats, notamment ceux obtenus par enquête longitudinale, constituent une première au Tchad. Toutefois, ils ne peuvent globalement être mieux appréciés qu'en les rapprochant des observations faites dans d'autres milieux mettant en œuvre des pratiques proches.

La proportion des mâles et des femelles dans les troupeaux porcins de la zone de N'Djaména a été conforme aux observations de Sana (1997), dans la zone dite de concentration englobant la zone de N'Djaména. Dans les élevages de porcs de race locale en zone périurbaine d'AbomeyCalavi et de Cotonou au Bénin, la taille moyenne des troupeaux est de 19 porcs dont 4,18 truies et 1,2 verrat en moyenne (Youssao et al., 2008). Le nombre élevé des femelles dans les troupeaux indique un souci des éleveurs de conserver les reproductrices afin d'assurer la reproductibilité du système. Par rapport aux résultats de Sana (1997), un accroissement des effectifs castrés est noté (7\% à 13\%), ce qui laisse croire à une tendance à l'engraissement de mâles castrés, mieux valorisés à la vente par les producteurs. Cependant, l'âge moyen à la castration dans notre site serait tardif, comparé à sa pratique entre 4 à 5 mois dans le Bassin arachidier sénégalais (Buldgen et al., 1994), 5 mois en ville et 6 mois dans les villages en Basse Casamance (Niang, 1997, cité par Ndiaye, 2007). La castration devrait intervenir entre 4 à 5 semaines, meilleure période pour améliorer la croissance des porcelets (Muys et al., 2003).

L'âge de sevrage en Basse Casamance intervient à 5 mois de façon naturelle et entre 2 à 3 mois s'il est provoqué (Missohou et al., 2001). Dans le Bassin arachidier, le sevrage a lieu vers 3 mois en ville et 2 mois dans les villages (Buldgen et al., 1994). Au Bénin, il est de 2 mois (Codjo, 2003, Youssao et al., 2009). En revanche, un intervalle compris entre 3 et 4 mois proche de nos résultats a été rapporté au Burkina (Kiendrébéogo, 2005). Dans les systèmes d'élevage traditionnel, le sevrage non provoqué serait tardif du fait de la non séparation entre les catégories d'animaux (Robert et al., 1999). Dans les zones tropicales, Muys et al. (2003) recommandent le sevrage à 2 mois. 
Les performances de reproduction des truies dans la zone de N'Djaména sont meilleures sur l'âge à la $1^{\text {ère }}$ mise-bas (MB), l'âge à la $1^{\text {ère }}$ saillie fécondante, la taille moyenne de la portée à la MB et au sevrage, par rapport aux observations faites en milieu rural du sud du Tchad (Mopaté et Koussou, 2003). En revanche, l'âge à la $1^{\text {ère }} \mathrm{MB}$ et d'entrée en reproduction sont identiques aux observations faites au Nord-Cameroun (Njoya et al., 1999). L'âge à la $1^{\text {ère }} \mathrm{MB}$ entre 16 et 17 mois et à la $1^{\text {ère }}$ saillie fécondante de 12 et 13 mois observés dans le Bassin arachidier sénégalais sont plus élevés (Buldgen et al., 1994). En revanche, la productivité numérique de 13 porcelets/ truie/an résultant de notre étude se situe dans l'intervalle de 12 à 14 rapportés par ces auteurs. La taille de la portée observée dans les élevages suivis a été proche des données d'enquête ponctuelle. Chez le porc de race locale du Bénin, la taille de la portée à la naissance est de 7,25 porcelets avec un taux de mortinatalité de $15,72 \%$ (Youssao et al., 2009). Les paramètres de reproduction dans la présente étude indiquent que le caractère quelque peu semi-intensif des productions animales en milieux urbains et périurbains de N'Djaména se distingue sur le plan des performances, des élevages extensifs ruraux. Les observations sur les races locales rustiques en France montrent que l'amélioration de la taille de portée peut venir d'une bonne conduite des élevages (Lebroue et al., 2000). Chez les porcs de la race locale du Bénin, l'amélioration du mode d'élevage (alimentation et suivi sanitaire) augmente significativement la taille de la portée de 5,33 à 8,8 porcelets par portée (Koutinhouin et al., 2009). L'évolution de la taille de portée selon le rang de mise-bas (RMB) indique la nécessité de ralentir la réforme des reproductrices dans les élevages. Plus le RMB augmente, plus la taille de portée s'améliore. La même observation est faite dans le Bassin arachidier du Sénégal sur un accroissement régulier de la portée de la $1^{\text {ère }}$ à la $3^{\text {ème }} \mathrm{MB}$ (Buldgen et al., 1994). Ce fait est exploité par les éleveurs Vietnamiens qui ne prononcent la réforme qu'au moment où le RMB des truies amorce une chute de la taille de portée vers 5 à 6 ans (Froelich, 1991). La réforme précoce à 3 ans dans notre zone expliquerait aussi l'absence observée des truies au-delà du $4^{\text {ème }}$ RMB. Des sorties précoces des truies par la vente sont très régulières après le $2^{\text {ème }} \mathrm{RMB}$ au Sénégal (Buldgen et al., 1994). Nos GMQ aussi bien chez les mâles que chez les femelles au $2^{\text {ème }}$ mois ont été proches des observations $(90,30 \mathrm{~g})$ faites au Burkina Faso (Zoungrana, 1990). La vitesse de croissance des femelles est plus élevée que celle des mâles pendant toute la période d'engraissement, avec des gains moyens quotidiens de 126,4 et $74 \mathrm{~g} / \mathrm{j}$ (Youssao et al., 2004). Selon cette même source, la croissance des porcelets est plus marquée dans les trois premiers mois d'engraissement que dans les trois derniers. Au-delà du matériel génétique exploité, ces différences de performances observées résultent pour l'essentiel des pratiques de production mises en œuvre par les producteurs.

Les aspects socio-économiques de cet élevage à N'Djaména font apparaître des avantages réels, tant sur le plan nutritionnel que économique. Cet élevage contribue avec les autres productions à cycles courts (viande de petits ruminants, produits avicoles et aquacoles), à l'approvisionnement des marchés urbains en produits animaux (Guerin et Faye, 1999). Le taux d'exploitation pourrait être amélioré par la réduction des pertes importantes dues surtout aux vols et aux mortalités. Les recettes de la vente font apparaître des marges bénéficiaires substantielles. Ces éléments confirment les observations sur les faibles coûts de production qui expliqueraient la compétitivité de la filière porcine au Tchad (Koussou et Duteurtre, 2002). Toutefois, les observations dans la zone de N'Djaména indiquent que des changements sont en train de s'opérer en matière de coûts de production de porcs. A cause des prix avantageux en ville, la marge brute annuelle des producteurs urbains a été deux fois plus élevée que celle des 
périurbains. Ces marges contribuent à l'amélioration des revenus des éleveurs de la zone. L'élevage de porcs comme toutes productions agricoles périurbaines est une nécessité qui s'impose quand les conditions économiques deviennent particulièrement difficiles (Sawio, 1995; Moustier, 1999 ; Moustier et de Bon, 2005). La majorité des éleveurs, notamment à la périphérie cultivaient les céréales. Malgré tout, l'essentiel des producteurs a utilisé les recettes de la vente des porcs en priorité pour s'approvisionner en céréales. Cela est un signe révélateur de l'insécurité alimentaire dans cette zone. Cet élevage apparaît comme une production meilleure à même de combler le déficit céréalier chronique et comme une activité pouvant contribuer à la lutte contre la pauvreté.

\section{Conclusion}

$\mathrm{La}$ structure des troupeaux, les performances de reproduction et celles socioéconomiques des élevages porcins de la zone de N'Djaména sont désormais connues. Il se dégage qu'une meilleure conduite des élevages et une sensibilisation des producteurs, sur la nécessité de ralentir les réformes des reproductrices dans les élevages permettraient d'améliorer la productivité des troupeaux. Les performances socioéconomiques de cette production font apparaitre des avantages réels, tant sur le plan nutritionnel qu'économique. Cet élevage apparaît comme une production meilleure à même d'améliorer l'alimentation carnée et les revenus des ménages. Ainsi, il contribue à la lutte contre la pauvreté dans la zone de N'Djaména. Il s'avère nécessaire de mettre en place une structure d'encadrement de la production et un suivi des élevages encadrés pour mesurer l'impact des interventions.

\section{REFERENCES}

Buldgen A, Piraux M, Dieng A, Schmit G, Compère R. 1994. Les élevages de porcs traditionnels $\mathrm{du}$ bassin arachidier sénégalais. Revue Mondiale de Zootechnie (RMZ), 80/81 (3-4) : 63 - 70.

Broutin C, Floquet A, Seck P, Tossou R, Edjah H. 2005. Agriculture et élevage face aux contraintes et opportunités de l'expansion urbaine: Exploration autour des villes de Thiès et Mboro au Sénégal, d'Abomey-Bohicon et Parakou au Bénin. Communication à l'atelier international «Agricultures Urbaines et Développement en Afrique Centrale et de l'Ouest », Yaoundé, Cameroun, 30/10 au 03/11/2005, 15 p. http://www.ecocite.org/ telechargement-fichiers/rapports/BroutinAgriculture-Yaounde.pdf

Codjo AB. 2003. Estimation des besoins énergétiques du porc local du Bénin en croissance entre 7 et $22 \mathrm{~kg}$ de poids vif. Tropicultura, 21 (2) : 56 - 60.

Froelich V. 1991. Essai d'analyse des systèmes d'élevage porcin. District de Nam Thanh - Delta du Fleuve Rouge. Montpellier, CNEARC. Mémoire de fin d'étude. 95 p.

Guerin H, Faye B. 1999. Spécificité de la problématique périurbaine pour les systèmes d'élevage. In : Moustier, Mbaye, De Bon, Guérin et Pages (éditeurs scientifiques), Agriculture périurbaine en Afrique subsaharienne. Actes de l'atelier, 20 - 24 avril 1998, Montpellier, France, Cirad, Coraf, pp. 43-49.

Kiendrébéogo T. 2005. Diagnostic des élevages porcins de la zone de BoboDioulasso: systèmes d'élevage et conditions technico-économiques de production. Mémoire de DEA en Gestion Intégrée des Ressources Naturelles (GIRN), option: Systèmes de Productions Animales, Université Polytechnique de Bobo-Dioulasso (UPB), Burkina Faso, 48 p. + annexes.

Koussou MO, Duteurtre G. 2002. Les facteurs de compétitivité de la filière porcine dans le bassin du Logone. Communication présentée au colloque «Systèmes agroalimentaires localisés _ Syal», 
Montpellier, Cirad, octobre 2002, 13 p. http://pigtrop.cirad.fr/fr/vie_scientifique/e conomie_Logone.htm

Koutinhouin GB, Youssao AKI, Toleba SS, Kpodekon TM, Ahounou GS, Bonou AG, Bessanvi J. 2009. Effet du mode d'élevage sur la prolificité des truies de race locale du Bénin et la viabilité de leurs porcelets, de la naissance au sevrage. Int. J. Biol. Chem. Sci., 3(4): 819-829.

Labroue F, Guillouet P, Marsac H, Boisseau C, Luquet M, Arrayet J, Martinat-Botte F, Terqui M. 2000. Etude des performances de reproduction de 5 races locales françaises. Journées de la Rech. Porcine $(J R P)$ en France, $32: 413-418$.

MEP (Ministère de l'Economie et du Plan), 2009. Deuxième recensement de la population et de l'habitat (RGPH2) : Résultats globaux. 88 p.

Missohou A, Niang M, Foucher H, Dieye PN. 2001. Les systèmes d'élevage porcin en Basse Casamance (Sénégal). Cahiers Agricultures, 10 : 405 - 408.

Mopaté LY, Koussou MO. 2003. L'élevage porcin, un élevage ignoré mais pourtant bien implanté dans les agro-sysèmes ruraux et périurbains du Tchad. In : (Jamin J. Y., Seyni Boukar L. et Floret C. éd. CD-ROM), Actes du colloque «Savanes africaines: des espaces en mutations, des acteurs face à des nouveaux défis », Garoua, Cameroun, 27 - $31 / 05 / 2002,9$ p.

Mopaté LY, Kaboré-Zoungrana CY, Gongnet PG. 2007. Dynamique des élevages porcins urbains : accroissement, caractéristiques des producteurs et des effectifs à N'Djaména (Tchad). Annales de l'Université de N’Djaména, série C, Sciences Exactes Appliquées et de la Santé, 2 : 75 - 91.

Mopaté LY, Koussou MO, Kaboré-Zoungrana CY. 2008. Consommateurs et consommation de la viande porcine en hors-foyer à N'Djaména (Tchad) (Tome 1). In Gouvernance et
Approvisionnement des Villes, Parrot L, Njoya A, Temple L, Assogba-Komlan F, Kahane R, Ba Diao M, Havard M (éds). Editions L'Harmattan ; 199 - 208.

Mopaté LY. 2008. Dynamique des élevages porcins et amélioration de la production en zones urbaine et périurbaine de la ville de N'Djaména (Tchad). Thèse de Doctorat Unique en Gestion Intégrée des ressources Naturelles, option: Productions Animales, Université Polytechnique de Bobo-Dioulasso (UPB), Burkina-Faso, p. 245.

Moustier P. 1999. Définitions et contours de l'agriculture périurbaine en Afrique au Subsaharienne. In : Moustier, Mbaye, De Bon, Guérin et Pages (éditeurs scientifiques), Actes de l'atelier «Agriculture périurbaine en Afrique subsaharienne », Cirad, Coraf, 20 - 24 avril 1998, Montpellier, France, p. 29 41.

Moustier P, de Bon H. 2005. Fonction d'alimentation et multifonctionnalité des agricultures périurbaines des villes du Sud. Les Cahiers de la Multifonctionnalité, 8 : 9 - 17.

Muys D, Westenbrink G, Meinderts J. 2003. L'élevage des porcs dans les zones tropicales Agrodoc1, Fondation Agromisa, Wageningen, Pays Bas, 86 p.

NDiaye RK. 2007. Epidémiologie de la peste porcine africaine (PPA) au Sénégal : Facteurs de risque en relation avec l'élevage porcin dans les régions de Fatick, Kaolak et Ziguinzor. Thèse de Doctorat de médecine vétérinaire $\mathrm{N}^{\circ} 4$, EISMV, Dakar, Sénégal, 157 p.

N'joya A, Awa ND, Ngo Tama AC. 1999. Système d'élevage porcin au Nord Cameroun: amélioration technicoéconomique. Rapport technique, IRAD de Garoua (Cameroun), 20 p.

Robert S, Weary D, Gonyou H. 1999. Sevrage précoce et bien-être des porcelets. Cahiers Agricultures, 8 : 471 - 476.

Sana SD. 1997. Enquête sur l'élevage porcin dans la zone d'intervention du Volet 
Animaux Villageois (V.A.V). Rapport d'enquête. Direction de l'élevage et des ressources animales (Déra), Projet appui au développement de l'économie Rurale (Ader), Volet animaux villageois (Vav), $35 \mathrm{p}$.

Sawio CJ. 1995. Qui sont les agriculteurs de Dar es-Salaam? In Faire campagne en ville : l'agriculture urbaine en Afrique de l'Est, Egziabher AG, Lee-Smith D, Maxwell DG, Memon PA, Mougeot LJA, Sawio CJ (éds). CRDI : Ottawa (Ontario), Canada ; $31-55$.

Youssao AKI, Mourot J, Gbangboché AB, Adéhan R, Akoutey A, Edénapko A. 2004. Influence du régime alimentaire sur les performances de croissance et les caractéristiques de la carcasse du porc local au Bénin. Revue Africaine de Santé et de Productions Animales (RASPA), 2(1) : $31-36$.

Youssao AKI, Koutinhouin GB, Kpodekon TM, Bonou AG, Adjakpa A, Dotcho CDG, Atodjinou FTR. 2008. Production porcine et ressources génétiques locales en zone périurbaine de Cotonou et d'Abomey-Calavi (Bénin). Rév. Elev. Méd. Vét. Pays Trop., 61(3) : $235-243$.

Youssao AKI, Koutinhouin GB, Kpodekon TM, Bonou A.G, Adjakpa A, Ahounou G.S, Mourot J. 2009. Performances zootechniques et aptitudes bouchères des porcs locaux au Sud du Bénin. Bull. Anim. Hlth. Prod. Afr., $57: 73-87$.

Zoungrana CY. 1990. Production laitière des truies et croissance des porcelets en milieu paysan. Bull. Anim. Hlth. Prod. Afr., 38, 245 - 251. 